YITP-13-80

\title{
Integrability of BPS equations in ABJM theory
}

\author{
Kazuhiro Saka $\oplus^{\dagger}$ and Seiji Terashima \\ ${ }^{\dagger}$ Department of Physical Sciences, Ritsumeikan University \\ Shiga 525-8577, Japan \\ ${ }^{\ddagger}$ Yukawa Institute for Theoretical Physics, Kyoto University \\ Kyoto 606-8502, Japan
}

\begin{abstract}
We investigate BPS equations which determine the configuration of an M2-M5 bound state preserving half of the supersymmetries in the ABJM theory. We argue that the BPS equations are classically integrable, showing that they admit a Lax representation. The integrable structure of the BPS equations is closely related to that of the Nahm equations. Using this relation we formulate an efficient way of constructing solutions of the BPS equations from those of the Nahm equations. As an illustration of our method, we construct explicitly the most general solutions describing two M2-branes suspended between two parallel M5-branes as well as two semi-infinite M2-branes ending on an M5-brane. These include previously unknown new solutions. We also discuss a reduction of the BPS equations in connection with the periodic Toda chain.
\end{abstract}

August 2013

\footnotetext{
${ }^{\dagger}$ ksakai@fc.ritsumei.ac.jp

${ }^{\ddagger}$ terasima@yukawa.kyoto-u.ac.jp
} 


\section{Introduction}

Towards understanding the still mysterious M-theory, the theories on M2-branes and M5-branes are expected to play crucial roles. Concerning M2-branes, we now have a strong candidate for the low energy effective theory on them, i.e. the AharonyBergman-Jafferis-Maldacena (ABJM) theory [1]. On the other hand, the low energy effective theory on multiple M5-branes is still unknown. The theory should be selfdual under a certain "electric-magnetic" duality, which may mean that construction of its action is unattainable by conventional field theory techniques.

A possible way to investigate M5-branes is to study M2-M5 bound states by means of the ABJM theory. In doing this one can circumvent the above difficulty because the ABJM action for $N$ M2-branes is an ordinary gauge field theory action with gauge group $\mathrm{U}(N) \times \mathrm{U}(N)$. Indeed, (classical) solutions describing M2-M5 bound states in the ABJM theory have been found [2 [5]. In particular, BPS equations obtained in [2, 3] are of fundamental importance. The equations can be considered as generalizations of the Basu-Harvey equations [6] and determine the configuration of an M2-M5 bound state preserving half of the supersymmetries in the ABJM theory. Explicit solutions of the BPS equations have been constructed [2,7,9].

It has been known [10] that BPS conditions for certain D2-D4 bound states are described by the Nahm equations. Because the M2-M5 bound state in M-theory is a counterpart of the D2-D4 bound state in string theory, the BPS equations in the ABJM theory should have a close connection with the Nahm equations. Indeed, it was found in [9] that one can always construct two sets of solutions of the Nahm equations from those of the BPS equations 1 The Nahm equations are classically integrable [16]. Moreover, based on the integrability one can systematically construct the Nahm data, i.e. the solutions of the Nahm equations [17, 18]. It is natural to expect that the present BPS equations are also integrable and one may be able to construct solutions systematically making use of the integrability.

In this paper we argue that the BPS equations in the ABJM theory are indeed classically integrable. We find a Lax representation for the BPS equations 2 As is expected, the integrable structure of the BPS equations is closely related to that of the Nahm equations. Using this relation we formulate an efficient way of constructing solutions of the BPS equations from those of the Nahm equations. As an illustra-

\footnotetext{
1 This kind of relation was first found in 11 for the Bagger-Lambert-Gustavsson theory [12 15].

${ }^{2}$ In [19], it was argued that the Basu-Harvey equation is integrable and a 3-bracket analog of the Lax representation was presented. (We would like to thank C. Sämann for informing us of the result.)
} 
tion, we construct explicitly the most general solutions describing two M2-branes suspended between two parallel M5-branes as well as two semi-infinite M2-branes ending on an M5-brane. These include previously unknown new solutions.

The organization of this paper is as follows. In section 2, we present the Lax representation of the BPS equations and introduce the auxiliary linear problem. We then clarify the relation between the integrable structure of the BPS equations and that of the Nahm equations. Using this relation we formulate a construction of solutions of the BPS equations starting from those of the Nahm equations. In section 3, we first study the auxiliary linear problem of the simple funnel-type solution. We then construct explicitly the most general solution describing two semi-infinite M2branes ending on an M5-brane, following the method described in section 2. We also construct the most general solution with $N=2$. This solution is expressed in terms of elliptic functions and describes two M2-branes suspended between two parallel M5-branes. In section 4, we discuss a reduction of the BPS equations in connection with the periodic Toda chain. Appendix A is a brief summary of the construction of the most general Nahm data with $N=2$. Conventions of special functions and some useful formulas are summarized in Appendix B.

\section{Integrability of BPS equations}

\subsection{BPS equations and Lax representation}

We are interested in BPS equations in the ABJM theory and their solutions. The equations are given by ordinary differential equations of the following form $3[2,3]$

$$
\dot{Y}^{a}=Y^{b} Y^{b \dagger} Y^{a}-Y^{a} Y^{b \dagger} Y^{b}
$$

Here $Y^{a}(s)(a=1,2)$ are $N \times N$ complex matrices and are functions in a real variable $s$. In this paper, a dot over letters stands for the derivative in $s$. Note that the above equations have an $\mathrm{SU}(N) \times \mathrm{SU}(N) \times \mathrm{SU}(2) \times \mathrm{U}(1)$ automorphism: If one transforms $Y^{a}$ as

$$
Y^{a} \rightarrow Y^{\prime a}=e^{i \varphi} \Lambda_{b}^{a} U Y^{b} V^{\dagger}
$$

with

$$
U, V \in \mathrm{SU}(N), \quad\left(\Lambda_{b}^{a}\right) \in \mathrm{SU}(2), \quad e^{i \varphi} \in \mathrm{U}(1),
$$

$Y^{\prime a}$ again satisfy the same equations as (2.1).

\footnotetext{
${ }^{3}$ The r.h.s. of these equations differ from those in [9] by an overall sign. Correspondingly, the sign of the M5-brane charge of the solutions in this paper is opposite to that in [9]. There are also several differences of conventions, which we will not mention hereafter.
} 
In this paper we argue that the BPS equations (2.1) are classically integrable 4 We find that the equations can be expressed in the form of the Lax equation:

$$
\dot{A}=[A, B]
$$

where

$$
\begin{aligned}
A(s ; \lambda) & =\left(\begin{array}{cc}
O & Y^{1}+\lambda Y^{2} \\
Y^{1 \dagger}-\lambda^{-1} Y^{2 \dagger} & O
\end{array}\right), \\
B(s ; \lambda) & =\left(\begin{array}{cc}
\lambda^{-1} Y^{1} Y^{2 \dagger}+\lambda Y^{2} Y^{1 \dagger} & O \\
O & \lambda Y^{1 \dagger} Y^{2}+\lambda^{-1} Y^{2 \dagger} Y^{1}
\end{array}\right) .
\end{aligned}
$$

Here $\lambda \in \mathbb{C}$ is the spectral parameter. There are actually various choice of Lax representations 5 We have chosen the above representation so that Lax operators admit several useful properties: The above Lax operators manifest two kinds of involution structures. First, they admit the following $\mathbb{Z}_{2}$-parity structure

$$
\{A, \Gamma\}=0, \quad[B, \Gamma]=0
$$

where

$$
\Gamma:=\left(\begin{array}{cc}
\mathbf{1}_{N} & 0 \\
0 & -\mathbf{1}_{N}
\end{array}\right)
$$

To see the other involution, let us introduce the following operation

$$
\mathcal{M}^{\star}(\lambda):=\mathcal{M}\left(-\bar{\lambda}^{-1}\right)^{\dagger}
$$

which we will call a star-involution. Here $\mathcal{M}$ is a matrix of any size and is a function in $\lambda$. Under this involution, the operators $A, B$ transform as

$$
A^{\star}=A, \quad B^{\star}=-B .
$$

Another useful property is the following relation

$$
B=\lambda \frac{\partial}{\partial \lambda} A^{2}
$$

We will make use of these structures to construct solutions.

\footnotetext{
${ }^{4}$ For the theory of classical integrable systems the reader should refer to textbooks, for example, [20, 21].

${ }^{5}$ For instance, one can take

$$
A^{\prime}=\left(\begin{array}{cc}
f(\lambda) \mathbf{1}_{N} & O \\
O & g(\lambda) \mathbf{1}_{N}
\end{array}\right) A, \quad B^{\prime}=B+h(A)
$$

with any nonzero functions $f, g, h$ as Lax operators. Such $A^{\prime}$ and $B^{\prime}$ reproduce the same BPS equations.
} 
As a general property of the Lax representation, it follows from (2.4) that the eigenvalues of $A$ are independent of $s$. Equivalently, the characteristic polynomial

$$
\begin{aligned}
P & :=\operatorname{det}\left(\eta \mathbf{1}_{2 N}-A\right) \\
& =\operatorname{det}\left[\eta^{2} \mathbf{1}_{N}-\left(Y^{1}+\lambda Y^{2}\right)\left(Y^{1 \dagger}-\lambda^{-1} Y^{2 \dagger}\right)\right] \\
& =\operatorname{det}\left[\eta^{2} \mathbf{1}_{N}-\left(Y^{1 \dagger}-\lambda^{-1} Y^{2 \dagger}\right)\left(Y^{1}+\lambda Y^{2}\right)\right] \quad(\eta \in \mathbb{C})
\end{aligned}
$$

is independent of $s$. It is convenient to introduce the notation

$$
\mu:=\eta^{2}
$$

The characteristic equation

$$
P(\mu, \lambda)=0
$$

gives the spectral curve of a given solution.

\subsection{Auxiliary linear problem}

The Lax equation is regarded as the compatibility condition of the following auxiliary linear problem

$$
\begin{aligned}
& A(s ; \lambda) \psi(s ; \lambda)=\eta(\lambda) \psi(s ; \lambda), \\
& B(s ; \lambda) \psi(s ; \lambda)=-\dot{\psi}(s ; \lambda) .
\end{aligned}
$$

The first equation describes an eigenvalue problem of the operator $A$, while the second one describes the evolution in $s$ of the eigenvector $\psi(s ; \lambda)$. Below we consider the case where the operator $A$ possesses $2 N$ linearly independent eigenvectors $\psi_{1}, \ldots, \psi_{2 N}$ with eigenvalues $\eta_{1}, \ldots, \eta_{2 N}$. The involution structure (2.8) implies that $A\left(\Gamma \psi_{m}\right)=$ $-\eta_{m}\left(\Gamma \psi_{m}\right)$, hence one can always choose the eigenvectors as

$$
\psi_{N+m}=\Gamma \psi_{m}, \quad \eta_{N+m}=-\eta_{m}, \quad m=1, \ldots, N
$$

If we introduce the notation

$$
\begin{aligned}
\Psi & :=\left(\psi_{1}, \ldots, \psi_{2 N}\right)=\left(\psi_{1}, \ldots, \psi_{N}, \Gamma \psi_{1}, \ldots, \Gamma \psi_{N}\right) \\
D & :=\operatorname{diag}\left(\eta_{1}, \ldots, \eta_{2 N}\right)=\operatorname{diag}\left(\eta_{1}, \ldots, \eta_{N},-\eta_{1}, \ldots,-\eta_{N}\right),
\end{aligned}
$$

the auxiliary linear problem can be expressed as

$$
\begin{aligned}
& A \Psi=\Psi D, \\
& B \Psi=-\dot{\Psi} .
\end{aligned}
$$


Since we have assumed that there exist $2 N$ linearly independent eigenvectors, $\Psi$ is a regular matrix at generic values of $s$ and $\lambda$. Therefore, one can invert (2.21) and express the operator $A$ as

$$
A(s ; \lambda)=\Psi(s ; \lambda) C(\lambda) \Psi^{\star}(s ; \lambda),
$$

where

$$
\begin{aligned}
C & :=D \mathcal{N}^{-1}, \\
\mathcal{N} & :=\Psi^{\star} \Psi .
\end{aligned}
$$

It is easy to verify that $\mathcal{N}$ and $C$ are independent of $s$ and are self-adjoint with respect to the star-involution.

The above structure implies that a generic solution of the original BPS equations can be expressed as a bilinear combination of $\Psi(s ; \lambda)$. As is well known, for a system with Lax representation there are several powerful techniques to restrict the form of $\Psi(s ; \lambda)$ and construct a class of general solutions. In the present case the situation is even better, due to the fact that the BPS equations are closely related to the Nahm equations. Solutions of the Nahm equations are well studied and using them one can easily determine the eigenvectors $\Psi(s ; \lambda)$, as we will explain in the next subsection.

\subsection{Relation to Nahm equations and construction of solutions}

Recall that the Nahm equations are given as

$$
\dot{T}^{I}=i \epsilon_{I J K} T^{J} T^{K}
$$

Here indices $I, J, K$ take values $1,2,3$ and $T^{I}$ are $N \times N$ hermitian matrices. It was found in [9] that one can always construct two sets of solutions of the Nahm equations from those of the BPS equations (2.1). Indeed, if $Y^{a}$ are solutions of (2.1), bilinear combinations

$$
T_{1}^{I}:=\left(\sigma^{I}\right)_{a b} Y^{a} Y^{b \dagger}, \quad T_{2}^{I}:=\left(\sigma^{I}\right)_{a b} Y^{b \dagger} Y^{a}
$$

both satisfy the Nahm equations (2.26). Here $\sigma^{I}$ denote Pauli matrices.

It has been known that the Nahm equations are classically integrable and admit a Lax representation [16]. Lax equations for the above $T_{\alpha}^{I}(\alpha=1,2)$ are written as

$$
\dot{A}_{\alpha}=\left[A_{\alpha}, B_{\alpha}\right]
$$


where

$$
\begin{aligned}
A_{\alpha} & :=T_{\alpha}^{3}+\frac{\lambda}{2}\left(T_{\alpha}^{1}-i T_{\alpha}^{2}\right)-\frac{1}{2 \lambda}\left(T_{\alpha}^{1}+i T_{\alpha}^{2}\right), \\
B_{\alpha} & :=\frac{\lambda}{2}\left(T_{\alpha}^{1}-i T_{\alpha}^{2}\right)+\frac{1}{2 \lambda}\left(T_{\alpha}^{1}+i T_{\alpha}^{2}\right) .
\end{aligned}
$$

As the reader may expect, these Lax operators are directly related to those for the BPS equations (2.5), (2.6). They are related in a remarkably simple way:

$$
A^{2}=\left(\begin{array}{cc}
A_{1} & 0 \\
0 & A_{2}
\end{array}\right), \quad B=\left(\begin{array}{cc}
B_{1} & 0 \\
0 & B_{2}
\end{array}\right) .
$$

This relation suggests that the Lax operator $A$ for the BPS equations is regarded as the "square root" of that for the Nahm equations. This is somewhat analogous to the relation between the Dirac operator and the Laplace operator.

The above relation implies an important fact: Any eigenvector of $A$ is divided into an eigenvector of $A_{1}$ and that of $A_{2}$ with a common eigenvalue. This fact provides us with an efficient way of constructing solutions of the BPS equations, as we explain below.

Suppose that we are given a pair of Nahm data $T_{\alpha}^{I}$ for which the Lax operators $A_{\alpha}(\alpha=1,2)$ have the same set of eigenvalues $\mu_{1}(\lambda), \ldots, \mu_{N}(\lambda)$. Put differently, the pair of Nahm data share the same spectral curve

$$
P(\mu, \lambda)=0
$$

with

$$
P=\operatorname{det}\left(\mu \mathbf{1}_{N}-A_{1}\right)=\operatorname{det}\left(\mu \mathbf{1}_{N}-A_{2}\right) .
$$

We assume that each of $A_{\alpha}$ has $N$ linearly independent eigenvectors. In this case, the eigenvalue problems of $A_{\alpha}$ can be expressed as

$$
A_{\alpha} \Psi_{\alpha}=\Psi_{\alpha} M
$$

with

$$
M=\operatorname{diag}\left(\mu_{1}, \ldots, \mu_{N}\right) .
$$

Here $\Psi_{\alpha}(s ; \lambda)$ are regular matrices at generic values of $s$ and $\lambda$. Since the Lax equations (2.28) hold, one can always normalize $\Psi_{\alpha}$ in such a way that they satisfy

$$
B_{\alpha} \Psi_{\alpha}=-\dot{\Psi}_{\alpha}
$$

By using this equation and $B_{\alpha}^{\star}=-B_{\alpha}$, one can verify that

$$
\mathcal{N}_{\alpha}:=\Psi_{\alpha}^{\star} \Psi_{\alpha}
$$


are independent of $s$.

As we mentioned above, if we express a solution (2.19) of the auxiliary linear problem (2.21), (2.22) as

$$
\Psi=\frac{1}{\sqrt{2}}\left(\begin{array}{cc}
\Psi_{1} & \Psi_{1} \\
\Psi_{2} & -\Psi_{2}
\end{array}\right)
$$

the submatrices $\Psi_{\alpha}$ are automatically solutions of the equations (2.34), (2.36) with $A_{\alpha}, B_{\alpha}$ given by (2.31). On the other hand, if we start from a given pair of Nahm data (sharing the same spectral curve) and put eigenvectors $\Psi_{\alpha}$ into the form (2.38), it does not always give a solution of the auxiliary linear problem of the BPS equations. Below let us examine under what conditions eigenvectors $\Psi_{\alpha}$ for given Nahm data generate a solution of the BPS equations.

The above $\Psi$ is associated with the eigenvalue matrix (2.20) of the form

$$
D=\left(\begin{array}{cc}
H & O \\
O & -H
\end{array}\right), \quad H=\operatorname{diag}\left(\eta_{1}, \ldots, \eta_{N}\right) .
$$

Since operators $A$ and $A_{\alpha}$ are related as in (2.31), their eigenvalues are related as

$$
H^{2}=M
$$

As we saw in the last subsection, the operator $A$ can be constructed from $\Psi$ as in (2.23). One could start from this general form, but here it is not difficult to guess the form of $A$ : One can check that the above $\Psi$ indeed gives the eigenvectors of the eigenvalue problem (2.21) if the operator $A$ is given as

$$
A=\left(\begin{array}{cc}
O & \Psi_{1} H \mathcal{N}_{2}^{-1} \Psi_{2}^{\star} \\
\Psi_{2} H \mathcal{N}_{1}^{-1} \Psi_{1}^{\star} & O
\end{array}\right) .
$$

In order for $A$ to have the property $A^{\star}=A$, it is required that

$$
H \mathcal{N}_{1}=\mathcal{N}_{2} H
$$

The evolution equation (2.22) automatically follows from (2.36). The remaining requirement is that the upper-right block of (2.41) is linear in $\lambda$, i.e.

$$
\frac{\partial^{2}}{\partial \lambda^{2}}\left[\Psi_{1} H \mathcal{N}_{2}^{-1} \Psi_{2}^{\star}\right]=0
$$

If both of the above conditions are satisfied, one obtains a consistent realization of the auxiliary linear problem of the BPS equations. The solutions $Y^{a}$ of the original BPS equations are then obtained explicitly as

$$
Y^{1}=\left.\Psi_{1} H \mathcal{N}_{2}^{-1} \Psi_{2}^{\star}\right|_{\lambda=0}, \quad Y^{2}=\left.\frac{\partial}{\partial \lambda}\left[\Psi_{1} H \mathcal{N}_{2}^{-1} \Psi_{2}^{\star}\right]\right|_{\lambda=0} .
$$


To sum up, we have seen that the solutions of the BPS equations can be constructed starting from the solutions of the Nahm equations. The construction of Nahm data has been extensively studied [17, 18]. Given a pair of Nahm data $T_{\alpha}^{I}$, it is in principle a straightforward task to compute eigenvectors $\Psi_{\alpha}$. The condition (2.42) can be easily satisfied: For instance, it is satisfied if we normalize $\Psi_{\alpha}$ as

$$
\mathcal{N}_{1}=\mathcal{N}_{2}=\operatorname{diag}\left(n_{1}(\lambda), \ldots, n_{N}(\lambda)\right)
$$

with some nonzero functions $n_{m}(\lambda)$. On the other hand, the condition (2.43) usually gives nontrivial constraints. To the best of our knowledge, we have to deal with this condition case by case. Nevertheless, the above procedure provides us with an efficient way of constructing a wide class of solutions of the BPS equations. In the next section, we will illustrate this construction by explicit examples.

\section{General solutions}

In this section we construct new solutions of the BPS equations following the method described in the last section. We first discuss the funnel-type solution with general $N$ and then focus on the general solutions with $N=2$. The solutions with $N=2$ are of fundamental importance, since a wide class of solutions with $N>2$ written in terms of elementary functions or elliptic functions are, up to the global symmetry transformation (2.2), direct sums of these solutions.

General solutions with $N=2$ are written in terms of elliptic functions. For the purpose of illustrating our method, however, it is enough to work on the semiinfinite solutions, which are written simply in terms of hyperbolic functions. We then generalize them to elliptic solutions and see that they indeed correspond to the most general Nahm data with $N=2$.

\subsection{Funnel-type solution and auxiliary linear problem}

Let us first consider the funnel-type solution [9]. In this case we already know the solution, but as a warm up exercise let us verify that the solution is indeed derived from the corresponding Nahm data.

The solution is given as

$$
Y^{1}=\sqrt{\frac{c}{1-e^{-2 x}}} G^{1}, \quad Y^{2}=\sqrt{\frac{c}{e^{2 x}-1}} G^{2},
$$

where

$$
x=c s, \quad c \geq 0
$$


and $G^{a}$ are constant $N \times N$ matrices satisfying

$$
-G^{a}=G^{b} G^{b \dagger} G^{a}-G^{a} G^{b \dagger} G^{b}
$$

One can take $G^{a}$ as

$$
\left(G^{1}\right)_{m n}=\sqrt{N-n} \delta_{m, n}, \quad\left(G^{2}\right)_{m n}=\sqrt{n} \delta_{m, n+1}
$$

Let us next introduce

$$
\tau_{1}^{i}:=\frac{1}{2}\left(\sigma^{i}\right)_{a b} G^{a} G^{b \dagger}, \quad \tau_{2}^{i}:=\frac{1}{2}\left(\sigma^{i}\right)_{a b} G^{b \dagger} G^{a} \quad(i=1,2,3,4),
$$

where $\sigma^{I}(I=1,2,3)$ are Pauli matrices and $\sigma^{4}=\mathbf{1}_{2}$. It follows from (3.3) that

$$
\left[\tau_{\alpha}^{I}, \tau_{\alpha}^{J}\right]=i \epsilon_{I J K} \tau_{\alpha}^{K}, \quad\left[\tau_{\alpha}^{I}, \tau_{\alpha}^{4}\right]=0
$$

In fact, $\tau_{1}^{I}$ and $\tau_{2}^{I}(I=1,2,3)$ are $\boldsymbol{N}$ and $\boldsymbol{N}-\mathbf{1} \oplus \mathbf{1}$ representations of $\operatorname{SU}(2)$, respectively. By using the relation (2.27), the corresponding Nahm data are obtained as

$$
T_{\alpha}^{1}=\frac{c}{\sinh x} \tau_{\alpha}^{1}, \quad T_{\alpha}^{2}=\frac{c}{\sinh x} \tau_{\alpha}^{2}, \quad T_{\alpha}^{3}=\frac{c}{\tanh x} \tau_{\alpha}^{3}+c \tau_{\alpha}^{4} .
$$

Let us now regard the above Nahm data as input and derive the solution of the BPS equation following the method explained in the last section. Here we first assume that $c>0$ and later comment on the limit $c \rightarrow 0$. The Lax operators for the Nahm data are written as

$$
A_{\alpha}=c\left(\frac{1}{\tanh x} \rho_{\alpha}^{3}+\rho_{\alpha}^{4}-\frac{i}{\sinh x} \rho_{\alpha}^{2}\right), \quad B_{\alpha}=\frac{c}{\sinh x} \rho_{\alpha}^{1},
$$

where

$$
\begin{array}{ll}
\rho_{\alpha}^{1}:=\frac{\lambda+\lambda^{-1}}{2} \tau_{\alpha}^{1}+\frac{\lambda-\lambda^{-1}}{2 i} \tau_{\alpha}^{2}, & \rho_{\alpha}^{2}:=-\frac{\lambda-\lambda^{-1}}{2 i} \tau_{\alpha}^{1}+\frac{\lambda+\lambda^{-1}}{2} \tau_{\alpha}^{2}, \\
\rho_{\alpha}^{3}:=\tau_{\alpha}^{3}, & \rho_{\alpha}^{4}:=\tau_{\alpha}^{4} .
\end{array}
$$

Clearly, $\rho_{\alpha}^{i}$ satisfy the same algebra (3.6) as $\tau_{\alpha}^{i}$ do. Using this fact, one can easily diagonalize $A_{\alpha}$ as

$$
A_{\alpha}=c\left(\tanh \frac{x}{2}\right)^{-\rho_{\alpha}^{1}}\left(\rho_{\alpha}^{3}+\rho_{\alpha}^{4}\right)\left(\tanh \frac{x}{2}\right)^{\rho_{\alpha}^{1}}
$$

One can check that

$$
\Psi_{\alpha}=\left(\tanh \frac{x}{2}\right)^{-\rho_{\alpha}^{1}}
$$


is a solution of the auxiliary linear problem (2.34), (2.36) with

$$
M=c\left(\rho_{\alpha}^{3}+\rho_{\alpha}^{4}\right)=c\left(G^{1}\right)^{2} .
$$

Observing that $\left(\rho_{\alpha}^{1}\right)^{\star}=-\rho_{\alpha}^{1}$, one finds that

$$
\mathcal{N}_{\alpha}=\mathbf{1}_{N}
$$

One can take $H=M^{1 / 2}$ as

$$
H=\sqrt{c} G^{1}
$$

Substituting these into the general expression (2.41), one obtains the explicit form of the Lax operator $A$. The results indeed takes the form (2.5) with the original solution (3.1).

In the limit $c \rightarrow 0$, the auxiliary linear problem becomes degenerate, i.e. the Lax operator $A$ does not have $2 N$ independent eigenvectors. Nevertheless, one finds that (3.1) does give a well-defined solution $Y^{a}=(2 s)^{-1 / 2} G^{a}$ in the limit $c \rightarrow 0$. In this way, it is often possible to obtain a solution even if the corresponding auxiliary linear problem is degenerate.

\subsection{Semi-infinite solutions}

In this subsection we construct the general BPS solution describing two semi-infinite M2-branes ending on an M5-brane. We set the M5-brane at $s=0$ and consider the solution over the semi-infinite line $s>0$. The solutions $Y^{a}(s)$ are regular for $s>0$ and diverge at $s=0$. For $s \rightarrow \infty$, they approach constant values, i.e.

$$
\dot{Y}^{a}(\infty)=0
$$

Using the BPS equations at $s=\infty$, one can check that $Y^{1}(\infty)$ and $Y^{2}(\infty)$ can be simultaneously diagonalized by a suitable transformation $Y^{a} \rightarrow U Y^{a} V^{\dagger}$ with $U, V \in \mathrm{SU}(2)$. Note that the values of diagonal elements of $Y^{a}(\infty)$ represent the locations of two M2-branes in the four directions tangent to the M5-brane.

When both of the matrices $Y^{1}(\infty)$ and $Y^{2}(\infty)$ are diagonal, the corresponding Nahm data $T_{\alpha}^{I}(\infty)$ are also diagonal and

$$
T_{1}^{I}(\infty)=T_{2}^{I}(\infty)
$$

It is shown in Appendix A that any Nahm data with $N=2$ can be expressed in the canonical form (A.6) by a suitable transformation. Nahm data of the form (A.6) 
which satisfy the above properties at $s=\infty$ are easily determined a: 6

$$
\begin{aligned}
T_{\alpha}^{1} & =\frac{c}{\sinh \left(x-x_{\alpha}\right)} \frac{\sigma^{1}}{2}+t^{1} \mathbf{1}_{2}, \quad T_{\alpha}^{2}=\frac{c}{\sinh \left(x-x_{\alpha}\right)} \frac{\sigma^{2}}{2}+t^{2} \mathbf{1}_{2}, \\
T_{\alpha}^{3} & =\frac{c}{\tanh \left(x-x_{\alpha}\right)} \frac{\sigma^{3}}{2}+t^{3} \mathbf{1}_{2} .
\end{aligned}
$$

Here $t^{I}, x_{\alpha}(\alpha=1,2)$ are real constants and

$$
x=c s, \quad c \geq 0 .
$$

Since the solutions $Y^{a}(s)$ are regular for $s>0$, both sets of Nahm data $T_{\alpha}^{I}$ have to be regular at least for $s>0$. The singular behavior of $Y^{a}(s)$ at $s=0$ implies that at least one set of the Nahm data are also singular at $s=0$. To satisfy these conditions, one can take

$$
x_{1}=0, \quad x_{2}=-l, \quad l \geq 0,
$$

without loss of generality. Note that one can take $T_{2}^{I}$ as constant solutions. This is realized as a special case where $l$ is sent to infinity.

The Lax operators are obtained as

$$
A_{\alpha}=\left(\tanh \frac{x-x_{\alpha}}{2}\right)^{-\rho^{1}} M\left(\tanh \frac{x-x_{\alpha}}{2}\right)^{\rho^{1}}
$$

with

$$
\begin{aligned}
M & =\left(\frac{c}{2} \sigma^{3}+t_{\lambda} \mathbf{1}_{2}\right) \\
\rho^{1} & =\frac{\lambda+\lambda^{-1}}{4} \sigma^{1}+\frac{\lambda-\lambda^{-1}}{4 i} \sigma^{2}=\frac{1}{2}\left(\begin{array}{cc}
0 & \lambda^{-1} \\
\lambda & 0
\end{array}\right), \\
t_{\lambda} & =t^{3}+\frac{\lambda}{2}\left(t^{1}-i t^{2}\right)-\frac{1}{2 \lambda}\left(t^{1}+i t^{2}\right) .
\end{aligned}
$$

The eigenvector matrices of $A_{\alpha}$ are given as

$$
\Psi_{\alpha}=\left(\tanh \frac{x-x_{\alpha}}{2}\right)^{-\rho^{1}} D_{\alpha},
$$

\footnotetext{
${ }^{6}$ To be precise, only one of the two sets of Nahm data can be transformed into the canonical form (A.6), because the $\mathrm{SO}(3)$ automorphism $T_{\alpha}^{I} \rightarrow L_{J}^{I} T_{\alpha}^{J}$ acts simultaneously on both $T_{1}^{I}$ and $T_{2}^{I}$. If we transform $T_{1}^{I}$ into the canonical form, the coefficients of Pauli matrices in $T_{2}^{I}$ are left with $\mathrm{SO}(3)$ degrees of freedom a priori. In the present case, however, only the 1-2 rotation of the $\mathrm{SO}(3)$ is compatible with the boundary condition (3.16). Furthermore, this degree of freedom can be removed by the $\mathrm{SU}(2)$ transformation $T_{2}^{I} \rightarrow V T_{2}^{I} V^{\dagger}$ because in the present case all the offdiagonal components of $T_{2}^{1}$ and $T_{2}^{2}$ are proportional to a single function $c / \sinh \left(x-x_{2}\right)$. As a result, one can take the two sets of Nahm data as in (3.17) without loss of generality.
} 
where $D_{\alpha}$ are diagonal matrices satisfying

$$
D_{\alpha}^{\star} D_{\alpha}=\mathbf{1}_{2}
$$

The above $\Psi_{\alpha}$ are normalized as

$$
\mathcal{N}_{\alpha}=\mathbf{1}_{2}
$$

We are now in a position to impose the condition (2.43). To do this, let us first evaluate the matrix

$$
\Psi_{1} H \mathcal{N}_{2}^{-1} \Psi_{2}^{\star}=\left(\tanh \frac{x-x_{1}}{2}\right)^{-\rho^{1}} D_{1} M^{1 / 2} D_{2}^{\star}\left(\tanh \frac{x-x_{2}}{2}\right)^{\rho^{1}}
$$

The matrix $D_{1} M^{1 / 2} D_{2}^{\star}$ is diagonal. We express it as

$$
D_{12}:=D_{1} M^{1 / 2} D_{2}^{\star}=\left(\begin{array}{cc}
\delta_{+}(\lambda) & 0 \\
0 & \delta_{-}(\lambda)
\end{array}\right) .
$$

By observing

$$
\left(\tanh \frac{x}{2}\right)^{ \pm \rho^{1}}=\frac{1}{\sqrt{2 \sinh x}}\left(\begin{array}{cc}
e^{x / 2} & \mp \lambda^{-1} e^{-x / 2} \\
\mp \lambda e^{-x / 2} & e^{x / 2}
\end{array}\right)
$$

and using the values of $x_{\alpha}$ specified in (3.19), the matrix (3.27) is evaluated as

$$
\begin{aligned}
& \Psi_{1} H_{\mathcal{N}_{2}^{-1} \Psi_{2}^{\star}} \\
& =\frac{1}{2 \sqrt{\sinh x \sinh (x+l)}}\left(\begin{array}{cc}
\delta_{+} e^{x+l / 2}-\delta_{-} e^{-x-l / 2} & \lambda^{-1}\left(-\delta_{+} e^{-l / 2}+\delta_{-} e^{l / 2}\right) \\
\lambda\left(\delta_{+} e^{l / 2}-\delta_{-} e^{-l / 2}\right) & -\delta_{+} e^{-x-l / 2}+\delta_{-} e^{x+l / 2}
\end{array}\right) .
\end{aligned}
$$

In order for the diagonal components of this matrix to be linear in $\lambda, \delta_{ \pm}(\lambda)$ have to be linear in $\lambda$. Moreover, in order for the off-diagonal components also to be linear in $\lambda, \delta_{ \pm}(\lambda)$ have to satisfy

$$
\delta_{+} e^{l / 2}-\delta_{-} e^{-l / 2}=\alpha, \quad-\delta_{+} e^{-l / 2}+\delta_{-} e^{l / 2}=\beta \lambda
$$

with some constants $\alpha, \beta \in \mathbb{C}$. By solving these constraints, $\delta_{ \pm}$are expressed as

$$
\delta_{ \pm}(\lambda)=\frac{e^{ \pm l / 2} \alpha+e^{\mp l / 2} \beta \lambda}{e^{l}-e^{-l}}
$$

Next, observe that

$$
D_{12}^{\star} D_{12}=M
$$


Together with (3.32), this condition implies that

$$
\begin{aligned}
& \alpha \bar{\alpha}=\sinh l\left(c+2 t^{3} \tanh l\right), \quad \alpha \bar{\beta}=2 \sinh ^{2} l\left(t^{1}+i t^{2}\right), \\
& \beta \bar{\beta}=\sinh l\left(c-2 t^{3} \tanh l\right) .
\end{aligned}
$$

From the consistency of these equations, parameters $l, c, t^{I}$ have to satisfy

$$
\left(t^{1}\right)^{2}+\left(t^{2}\right)^{2}+\frac{\left(t^{3}\right)^{2}}{\cosh ^{2} l}=\frac{c^{2}}{4 \sinh ^{2} l} .
$$

This is solved as

$$
t^{1}=\frac{c}{2 \sinh l} n_{1}, \quad t^{2}=\frac{c}{2 \sinh l} n_{2}, \quad t^{3}=\frac{c}{2 \tanh l} n_{3}
$$

with $\boldsymbol{n}=\left(n_{1}, n_{2}, n_{3}\right)^{\mathbf{T}}$ being a three-dimensional unit vector. We parametrize it by

$$
\left(n_{1}, n_{2}, n_{3}\right)=(\sin \theta \cos \phi, \sin \theta \sin \phi, \cos \theta)
$$

with $0 \leq \theta \leq \pi, 0 \leq \phi<2 \pi$. By using this parametrization, $\alpha, \beta$ are solved as

$$
\alpha=\sqrt{2 c \sinh l} \cos \frac{\theta}{2} e^{i \chi+i \phi}, \quad \beta=\sqrt{2 c \sinh l} \sin \frac{\theta}{2} e^{i \chi}
$$

with $\chi \in \mathbb{R}$. The common phase factor $e^{i \chi}$ will eventually become an overall phase factor of the solution. It can be removed by the $\mathrm{U}(1)$ rotation $Y^{a} \rightarrow e^{-i \chi} Y^{a}$. Finally, using (2.44) one obtains

$$
\begin{aligned}
& Y^{1}=\sqrt{\frac{c}{2 \sinh l \sinh x \sinh (x+l)}}\left(\begin{array}{cc}
\sinh (x+l) \cos \frac{\theta}{2} e^{i \phi} & \sinh l \sin \frac{\theta}{2} \\
0 & \sinh x \cos \frac{\theta}{2} e^{i \phi}
\end{array}\right), \\
& Y^{2}=\sqrt{\frac{c}{2 \sinh l \sinh x \sinh (x+l)}}\left(\begin{array}{cc}
\sinh x \sin \frac{\theta}{2} & 0 \\
\sinh l \cos \frac{\theta}{2} e^{i \phi} & \sinh (x+l) \sin \frac{\theta}{2}
\end{array}\right) \text {. }
\end{aligned}
$$

The funnel-type solution in the last subsection is obtained by setting $\theta=\phi=0$ and taking the limit $l \rightarrow \infty$.

By construction this solution serves as the general solution with $N=2$ satisfying the semi-infinite boundary condition, up to translation in $x$ and the automorphism (2.2). The solution possesses four free parameters $c, l, \theta, \phi$. The number of free parameters coincides with that of the most general semi-infinite solution (3.17) of the Nahm equations: The solution (3.17) also possesses four free parameters $c$ and $t^{I}$, up to translation in $x$ and the $\mathrm{SU}(2) \times \mathrm{SO}(3)$ automorphism. It is interesting that one of the two sets of Nahm data $T_{\alpha}^{I}(\alpha=1,2)$ essentially determine the form of $Y^{a}$ as well as the other set of Nahm data. In particular, the distance of two singularities 
$l=x_{1}-x_{2}$ is uniquely determined if $c$ and $t^{I}$ are specified. From the point of view of M-theory, the coincidence of the degrees of freedom is regarded as the correspondence of the numbers of moduli between M2-M5 bound states and D2-D4 bound states.

The above number of free parameters is also consistent with the moduli counting in the ABJM theory. As we mentioned in the beginning of this subsection, $Y^{1}(\infty)$ and $Y^{2}(\infty)$ are simultaneously diagonalized by an $\mathrm{SU}(2) \times \mathrm{SU}(2)$ automorphism transformation $Y^{a} \rightarrow U Y^{a} V^{\dagger}$. The values of diagonal elements of $Y^{a}(\infty)$ give eight real moduli, which represent the locations of two M2-branes in the four directions tangent to the M5-brane. Four of the eight moduli correspond to the remaining $\mathrm{SU}(2) \times \mathrm{U}(1)$ part of the automorphism (2.2), i.e. the global symmetry of the solutions. The other four moduli are identified with the above $c, l, \theta, \phi$ which characterize the "shape" of the solutions. Notice that in the ABJM theory the above U(1) has to be regarded as a part of moduli rather than a gauge degree of freedom [1].

\subsection{General elliptic solutions}

The general semi-infinite solution (3.39) constructed in the last subsection can be expressed as

$$
\begin{aligned}
Y^{1} & =\frac{1}{2}\left(f_{1} \sin \frac{\theta}{2} \sigma^{1}+f_{2} \sin \frac{\theta}{2} i \sigma^{2}+f_{3} e^{i \phi} \cos \frac{\theta}{2} \sigma^{3}-f_{0} e^{i \phi} \cos \frac{\theta}{2} \mathbf{1}_{2}\right), \\
Y^{2} & =\frac{1}{2}\left(f_{1} e^{i \phi} \cos \frac{\theta}{2} \sigma^{1}-f_{2} e^{i \phi} \cos \frac{\theta}{2} i \sigma^{2}-f_{3} \sin \frac{\theta}{2} \sigma^{3}-f_{0} \sin \frac{\theta}{2} \mathbf{1}_{2}\right),
\end{aligned}
$$

where $\sigma^{I}$ are Pauli matrices and

$f_{1}=f_{2}=\sqrt{\frac{c \sinh l}{2 \sinh x \sinh (x+l)}}, \quad f_{3}=\frac{\cosh (x+l / 2)}{\cosh (l / 2)} f_{1}, \quad f_{0}=-\frac{\sinh (x+l / 2)}{\sinh (l / 2)} f_{1}$.

As wee see below, making use of this expression one can construct the general solution of the BPS equations for $N=2$ without repeating the procedure in the last subsection.

Let us first note that the above $f_{i}(s)$ are real functions and satisfy differential equations

$$
\dot{f}_{i}=f_{j} f_{k} f_{l}
$$

where the values of $i, j, k, l$ are taken to be all distinct. Interestingly, the differential equations (3.42) (and the restriction that $f_{i}$ are real functions) are sufficient for $Y^{a}(s)$ of the form (3.40) to satisfy the BPS equations (2.1). This means that one immediately obtains more general solutions of the BPS equations by merely finding 
more general solutions of (3.42). Observing that $f_{i}^{2}-f_{j}^{2}$ are constants, One can reduce (3.42) to a single first-order differential equation and construct the general solution. In fact, the general solution of (3.42) has already been obtained in [9]. In what follows, we present the solution in several new expressions. These new expressions are certainly useful for a clear understanding of the structure of the solution.

A sufficiently general solution of (3.42), which contains four independent parameters, is given by

$$
f_{i}=\frac{\vartheta_{i+1}(u)}{\vartheta_{i+1}\left(u_{*}\right)} \sqrt{\frac{\pi}{2 \omega_{1}} \frac{\vartheta_{1}\left(u_{*}\right) \vartheta_{2}\left(u_{*}\right) \vartheta_{3}\left(u_{*}\right) \vartheta_{4}\left(u_{*}\right)}{\vartheta_{1}\left(u_{*}+u\right) \vartheta_{1}\left(u_{*}-u\right)}} \quad(i=0,1,2,3) .
$$

Here $\vartheta_{i+1}(u):=\vartheta_{i+1}(u, \tau)$ are Jacobi theta functions (see Appendix B) and the variable $u$ is defined as

$$
u=\frac{s-s_{0}}{2 \omega_{1}} .
$$

This solution contains four parameters

$$
s_{0} \in \mathbb{R}, \quad 0<u_{*}<\frac{1}{2}, \quad \omega_{1} \in \mathbb{R}_{>0}, \quad \tau \in i \mathbb{R}_{>0} .
$$

The solution is defined over the region

$$
-u_{*}<u<u_{*}
$$

At each boundary of this region $f_{i}$ diverge. This implies that M2-branes are bounded by an M5-brane located at each of these boundaries. Thus, the present solution describes two M2-branes suspended between two parallel M5-branes.

Just outside this region the above $f_{i}$ become imaginary. However, slightly modifying them as

$$
\tilde{f}_{i}=(-1)^{\delta_{1, i}} \frac{\vartheta_{i+1}(u)}{\vartheta_{i+1}\left(u_{*}\right)} \sqrt{\frac{\pi}{2 \omega_{1}} \frac{\vartheta_{1}\left(u_{*}\right) \vartheta_{2}\left(u_{*}\right) \vartheta_{3}\left(u_{*}\right) \vartheta_{4}\left(u_{*}\right)}{\vartheta_{1}\left(u+u_{*}\right) \vartheta_{1}\left(u-u_{*}\right)}} \quad(i=0,1,2,3),
$$

one again obtains a set of real functions which satisfy the differential equations (3.42). This solution is now defined over the region

$$
u_{*}<u<1-u_{*} \text {. }
$$

The above $f_{i}$ and $\tilde{f}_{i}$ satisfy

$$
\begin{array}{rll}
f_{1} \geq f_{2} \geq f_{3}>-f_{0}>0 & \text { for } & -u_{*}<u<0, \\
f_{1} \geq f_{2} \geq f_{3}>f_{0}>0 & \text { for } & 0<u<u_{*}, \\
\tilde{f}_{0} \geq \tilde{f}_{3} \geq \tilde{f}_{2}>-\tilde{f}_{1}>0 & \text { for } & u_{*}<u<\frac{1}{2}, \\
\tilde{f}_{0} \geq \tilde{f}_{3} \geq \tilde{f}_{2}>\tilde{f}_{1}>0 & \text { for } & \frac{1}{2}<u<1-u_{*} .
\end{array}
$$


Any permutation of $f_{i}$ and/or overall sign change of even number of $f_{i}$ again give another solution to the equations (3.42), where the new $f_{i}$ satisfy different inequalities from (3.49). The same holds for $\tilde{f}_{i}$. All these constitute the general solution of (3.42).

In the following, let us concentrate on the solution (3.43) and study its properties. The solution is also concisely expressed in terms of Weierstrass elliptic functions (see Appendix B) as

$$
f_{0}=\left(\frac{\wp_{1}\left(s_{*}\right) \wp_{2}\left(s_{*}\right) \wp_{3}\left(s_{*}\right)}{\wp\left(s-s_{0}\right)-\wp\left(s_{*}\right)}\right)^{1 / 2}, \quad f_{I}=\frac{\wp_{I}\left(s-s_{0}\right)}{\wp_{I}\left(s_{*}\right)} f_{0} \quad(I=1,2,3),
$$

where

$$
s_{*}=2 \omega_{1} u_{*}, \quad 0<s_{*}<\omega_{1} .
$$

This solution is defined over the region $s_{0}-s_{*}<s<s_{0}+s_{*}$ and the branch of $f_{0}$ is taken in such a way that $f_{0} \gtrless 0$ for $0 \lessgtr s-s_{0} \lessgtr \pm s_{*}$.

As mentioned previously, $f_{I}^{2}-f_{0}^{2}$ are constants. These constants are expressed as

$$
\begin{aligned}
f_{I}^{2}-f_{0}^{2} & =\frac{\pi \vartheta_{I+1}^{2}}{2 \omega_{1}} \frac{\vartheta_{J+1}\left(u_{*}\right) \vartheta_{K+1}\left(u_{*}\right)}{\vartheta_{1}\left(u_{*}\right) \vartheta_{I+1}\left(u_{*}\right)} \\
& =\frac{\wp_{J}\left(s_{*}\right) \wp_{K}\left(s_{*}\right)}{\wp_{I}\left(s_{*}\right)} \\
& =: a_{I}^{2} \quad\left(a_{I}>0\right),
\end{aligned}
$$

where $I, J, K$ are any permutation of $1,2,3$. It can be checked that

$$
a_{1} \geq a_{2} \geq a_{3}>0
$$

Instead of $\omega_{1}, \tau, s_{*}, s_{0}$, one could use $a_{1}, a_{2}, a_{3}, s_{0}$ as free parameters. Using Jacobi elliptic functions, one can express the solution (3.43) as

$$
\begin{aligned}
f_{0} & =\frac{a_{3} \operatorname{sn} x}{\sqrt{\mathrm{sn}^{2} x_{*}-\mathrm{sn}^{2} x}}, & f_{1} & =\frac{a_{1} \operatorname{sn} x_{*} \operatorname{cn} x}{\sqrt{\mathrm{sn}^{2} x_{*}-\mathrm{sn}^{2} x}}, \\
f_{2} & =\frac{a_{2} \operatorname{sn} x_{*} \operatorname{dn} x}{\sqrt{\mathrm{sn}^{2} x_{*}-\mathrm{sn}^{2} x}}, & f_{3} & =\frac{a_{3} \operatorname{sn} x_{*}}{\sqrt{\mathrm{sn}^{2} x_{*}-\mathrm{sn}^{2} x}},
\end{aligned}
$$

where

$$
x=c\left(s-s_{0}\right), \quad c=a_{2} \sqrt{a_{1}^{2}-a_{3}^{2}}, \quad \text { sn } x_{*}=\sqrt{1-\frac{a_{3}^{2}}{a_{1}^{2}}} .
$$

By using the relation (2.27), the Nahm data corresponding to $Y^{a}(s)$ of the form (3.40) are obtained as

$$
T_{\alpha}^{I}=\left((-1)^{\alpha-1} f_{J} f_{K}-f_{I} f_{0}\right) \frac{\sigma^{I}}{2}+\frac{n_{I}}{4}\left(f_{I}^{2}-f_{J}^{2}-f_{K}^{2}+f_{0}^{2}\right) \mathbf{1}_{2},
$$


where $I, J, K$ are any permutation of $1,2,3$ and $n_{I}$ are given as in (3.37). Substituting (3.51) for $f_{i}$, the corresponding Nahm data are obtained as

$$
T_{\alpha}^{I}=\wp_{I}\left(s-s_{\alpha}\right) \frac{\sigma^{I}}{2}+\frac{n_{I}}{4}\left(a_{I}^{2}-a_{J}^{2}-a_{K}^{2}\right) \mathbf{1}_{2}
$$

with

$$
s_{1}=s_{0}-s_{*}, \quad s_{2}=s_{0}+s_{*}
$$

and $a_{I}$ given as in (3.53). One can also express these Nahm data in terms of either Jacobi theta functions or Jacobi elliptic functions: This is done by using

$$
\wp_{I}(\tilde{s})=\frac{\pi \vartheta_{J+1} \vartheta_{K+1}}{2 \omega_{1}} \frac{\vartheta_{I+1}(u)}{\vartheta_{1}(u)}
$$

with $u=\tilde{s} / 2 \omega_{1}$ or

$$
\wp_{1}(\tilde{s})=c \frac{\operatorname{cn} x}{\operatorname{sn} x}, \quad \wp_{2}(\tilde{s})=c \frac{\operatorname{dn} x}{\operatorname{sn} x}, \quad \wp_{3}(\tilde{s})=c \frac{1}{\operatorname{sn} x}
$$

with $x=c \tilde{s}=a_{2} \sqrt{a_{1}^{2}-a_{3}^{2}} \tilde{s}$, respectively.

Clearly, the above Nahm data are the most general solution to the Nahm equations with $N=2$. Up to the $\mathrm{SU}(2) \times \mathrm{SO}(3)$ automorphism, either of the two sets of Nahm data possess six free parameters $x_{\alpha}, \omega_{1}, \omega_{3}, t^{I}$. These parameters completely determine the six free parameters of $Y^{a}(s)$ with $N=2$. Thus, we again find that one of the two sets of Nahm data essentially determine a solution of the BPS equations.

Finally, let us see some particular limits of the solution. The Weierstrass $\wp-$ function is doubly periodic with respect to fundamental periods $2 \omega_{1}, 2 \omega_{3}$. (Recall that $\tau=\omega_{3} / \omega_{1}$ and here we take $\omega_{1} \in \mathbb{R}_{>0}$ and $\omega_{3} \in i \mathbb{R}_{>0}$.) One obtains rational solutions by sending either of the periods to infinity. If we take the limit $\omega_{3} \rightarrow i \infty$ (i.e. $\tau \rightarrow i \infty$ ), we obtain

$$
f_{0}=\frac{\sin x}{\sin x_{*}} f_{2}, \quad f_{1}=\frac{\cos x}{\cos x_{*}} f_{2}, \quad f_{2}=f_{3}=\sqrt{\frac{c \sin x_{*} \cos x_{*}}{\sin \left(x_{*}+x\right) \sin \left(x_{*}-x\right)}},
$$

where

$$
x=c\left(s-s_{0}\right), \quad x_{*}=c s_{*}, \quad c=\frac{\pi}{2 \omega_{1}} .
$$

On the other hand, if we take the limit $\omega_{1} \rightarrow \infty$ (i.e. $\tau \rightarrow i 0$ ), we obtain

$$
f_{0}=\frac{\sinh x}{\sinh x_{*}} f_{1}, \quad f_{3}=\frac{\cosh x}{\cosh x_{*}} f_{1}, \quad f_{1}=f_{2}=\sqrt{\frac{c \sinh x_{*} \cosh x_{*}}{\sinh \left(x_{*}+x\right) \sinh \left(x_{*}-x\right)}}
$$


where

$$
x=c\left(s-s_{0}\right), \quad x_{*}=c s_{*}, \quad c=\frac{\pi i}{2 \omega_{3}} .
$$

Note that $\omega_{3} / i \in \mathbb{R}_{>0}$. Note also that the semi-infinite solution is obtained from $\tilde{f}_{i}$ in the limit $\omega_{1} \rightarrow \infty$.

\section{Reduction in connection with periodic Toda chain}

It has been known that the Nahm equations reduce to Toda molecule equations [22]. Here we consider the reduction to the differential equations for the periodic Toda chain [23] as an example and present the corresponding reduction of the BPS equations. Interestingly, the reduction takes a remarkably simple form in terms of the BPS equations.

Let us make an ansatz of matrices $Y^{a}$ as follows:

$$
\left(Y^{1}\right)_{m n}=g_{m}(s) \delta_{m, n}, \quad\left(Y^{2}\right)_{m n}=h_{n}(s) \delta_{m, n+1},
$$

where $m, n=1, \ldots, N$. In this section the values of indices are identified $\bmod N$. By using a constant $\mathrm{SU}(N) \times \mathrm{SU}(N) \times \mathrm{U}(1)$ rotation one can take all $g_{m}, h_{m}$ to be real functions without loss of generality. The BPS equations (2.1) in this case become

$$
\begin{aligned}
& \dot{g}_{m}=\left(h_{m-1}^{2}-h_{m}^{2}\right) g_{m} \\
& \dot{h}_{m}=\left(g_{m+1}^{2}-g_{m}^{2}\right) h_{m} .
\end{aligned}
$$

Next, let us introduce

$$
\begin{array}{ll}
a_{m}:=g_{m+1} h_{m}, \quad & \tilde{a}_{m}:=g_{m} h_{m}, \\
b_{m}:=g_{m}^{2}-h_{m}^{2}, & \tilde{b}_{m}:=g_{m}^{2}-h_{m-1}^{2} .
\end{array}
$$

These particular combinations satisfy the differential equations of the periodic Toda chain with imaginary coupling

$$
\begin{aligned}
& \dot{a}_{m}=a_{m}\left(b_{m+1}-b_{m}\right), \\
& \dot{b}_{m}=-2\left(a_{m}^{2}-a_{m-1}^{2}\right) .
\end{aligned}
$$

The same equations hold for $\tilde{a}_{m}, \tilde{b}_{m}$.

The simplest case is the reduction of the form (4.1) with $N=2$. In this case, the general solution is given by the general elliptic solution (3.40) with $\theta=\phi=0$ and $f_{i}$ given in the last section. 


\section{Discussion}

In this paper we have investigated classical integrability of BPS equations in the ABJM theory. The integrable structure of the BPS equations is closely related to that of the Nahm equations. Making use of this fact, we have formulated an efficient way of constructing solutions of the BPS equations from those of the Nahm equations. As an illustration, we have constructed explicitly the most general solutions describing two M2-branes suspended between two parallel M5-branes as well as two semi-infinite M2-branes ending on an M5-brane.

We have observed that solutions of the BPS equations are uniquely determined if the corresponding Nahm data are given. It would be of interest if one could formulate a more direct way of constructing solutions of the BPS equations from given Nahm data.

We have elucidated that the number of free parameters of the semi-infinite solutions is in perfect agreement with the moduli counting of the ABJM theory. On the other hand, we have not yet found a clear explanation of the physical meaning of the number of free parameters in the general solution from the point of view of M-theory. It is interesting to clarify the structure of the moduli space of solutions and interpret it in the context of M-theory.

We have focused on the BPS equations which determine the "flat" M2-branes suspended between parallel M5-branes. It would be of great interest if BPS equations corresponding to other configurations could also exhibit a similar integrable structure.

\section{Acknowledgments}

The authors would like to thank S. Kakei, K. Hosomichi, M. Hamanaka, S. Lee, D. Muranaka, T. Nosaka, K. Takasaki, T. Takebe for discussions. The works of K.S. and S.T. are supported in part by Grant-in-Aid for Scientific Research from the Japan Ministry of Education, Culture, Sports, Science and Technology (MEXT).

\section{A. General Nahm data with $N=2$}

In this appendix we present a construction of the general solution of the Nahm equations (2.26) with $N=2$. The Nahm data $T^{I}(I=1,2,3)$ are hermitian matrices. For $N=2$, any $T^{I}(s)$ can be expressed as

$$
T^{I}(s)=F_{J}^{I}(s) \frac{\sigma^{J}}{2}+t^{I}(s) \mathbf{1}_{2}
$$


where $F_{J}^{I}, t^{I}$ are real functions and $\sigma^{J}$ are Pauli matrices. The Nahm equations imply that

$$
\sum_{I=1}^{3} F_{J}^{I} F_{K}^{I}=\text { const. }, \quad \sum_{I=1}^{3} F^{J}{ }_{I} F^{K}{ }_{I}=\text { const. } \quad \text { for } \quad J \neq K
$$

and

$$
t^{I}=\text { const. }
$$

Recall that the Nahm equations are invariant under the transformations $T^{I} \rightarrow L_{J}^{I} T^{J}$ and $T^{I} \rightarrow U T^{I} U^{\dagger}$, where $\left(L_{J}^{I}\right)$ and $U$ are constant $\mathrm{SO}(3)$ and $\mathrm{SU}(2)$ matrices, respectively. Using these transformations, one can transform $F^{I}{ }_{J}$ at a fixed value of $s$ into the form

$$
F_{J}^{I}=F^{I} \delta_{J}^{I}
$$

At this value of $s$,

$$
F_{J}^{I}=0 \quad \text { for } \quad I \neq J .
$$

It then follows from (A.2) that (A.5) hold for any value of $s$. Thus, without loss of generality one can assume the form of the Nahm data to be

$$
T^{I}(s)=F^{I}(s) \frac{\sigma^{I}}{2}+t^{I} \mathbf{1}_{2}
$$

where $F^{I}(s)$ are real functions and $t^{I}$ are real constants.

It follows from the Nahm equations that $F^{I}(s)$ satisfy the following differential equations

$$
\dot{F}^{I}=-F^{J} F^{K}
$$

where $I, J, K$ are any permutation of $1,2,3$. The general solution to this equation is most concisely expressed in terms of Weierstrass elliptic functions as

$$
F^{I}=\wp_{I}\left(s-s_{0} ; 2 \omega_{1}, 2 \omega_{3}\right)
$$

with

$$
s_{0} \in \mathbb{R}, \quad \omega_{1} \in \mathbb{R}_{>0}, \quad \omega_{3} \in i \mathbb{R}_{>0} .
$$

It is also possible to express $F^{I}$ in terms of Jacobi theta functions or Jacobi elliptic functions by using (3.60) or (3.61), respectively. Any permutation of $F^{I}$ and/or overall sign change of even number of $F^{I}$ again give another solution to the equations. 


\section{B. Conventions of special functions}

The Jacobi theta functions are defined as

$$
\begin{aligned}
& \vartheta_{1}(z, \tau)=i \sum_{n \in \mathbb{Z}}(-1)^{n} y^{n-1 / 2} q^{(n-1 / 2)^{2} / 2} \\
& \vartheta_{2}(z, \tau)=\sum_{n \in \mathbb{Z}} y^{n-1 / 2} q^{(n-1 / 2)^{2} / 2} \\
& \vartheta_{3}(z, \tau)=\sum_{n \in \mathbb{Z}} y^{n} q^{n^{2} / 2} \\
& \vartheta_{4}(z, \tau)=\sum_{n \in \mathbb{Z}}(-1)^{n} y^{n} q^{n^{2} / 2}
\end{aligned}
$$

where $y=e^{2 \pi i z}, q=e^{2 \pi i \tau}$. We often use the following abbreviated notation

$$
\vartheta_{k}(z):=\vartheta_{k}(z, \tau), \quad \vartheta_{k}:=\vartheta_{k}(0, \tau)
$$

The Weierstrass $\wp$-function is defined as

$$
\wp(z)=\wp\left(z ; 2 \omega_{1}, 2 \omega_{3}\right):=\frac{1}{z^{2}}+\sum_{(m, n) \in \mathbb{Z}_{\neq(0,0)}^{2}}\left[\frac{1}{\left(z-\Omega_{m, n}\right)^{2}}-\frac{1}{\Omega_{m, n}^{2}}\right],
$$

where $\Omega_{m, n}=2 m \omega_{1}+2 n \omega_{3}$. We also introduce the following notation

$$
e_{I}:=\wp\left(\omega_{I}\right) \quad(I=1,2,3),
$$

with

$$
\omega_{1}+\omega_{2}+\omega_{3}=0, \quad \frac{\omega_{3}}{\omega_{1}}=\tau .
$$

The functions $\wp_{I}(z)=\wp_{I}\left(z ; 2 \omega_{1}, 2 \omega_{3}\right)$ are then defined as

$$
\wp_{I}(z):=\left(\wp(z)-e_{I}\right)^{1 / 2}
$$

where the branch of the square root is chosen so that $\wp_{I}(z)=1 / z+\mathcal{O}(z)$. Let us present some useful formulas:

$$
\begin{aligned}
\wp^{\prime}(z) & =-2 \wp_{1}(z) \wp_{2}(z) \wp_{3}(z), \\
\wp_{I}(z+w) & =-\frac{\wp_{I}(z) \wp_{J}(w) \wp_{K}(w)-\wp_{I}(w) \wp_{J}(z) \wp_{K}(z)}{\wp(z)-\wp(w)},
\end{aligned}
$$

where $I, J, K$ are any permutation of $1,2,3$. The following relations are also useful:

$$
\wp_{1}(s)=\sqrt{e_{1}-e_{3}} \frac{\operatorname{cn} x}{\operatorname{sn} x}, \quad \wp_{2}(s)=\sqrt{e_{1}-e_{3}} \frac{\operatorname{dn} x}{\operatorname{sn} x}, \quad \wp_{3}(s)=\sqrt{e_{1}-e_{3}} \frac{1}{\operatorname{sn} x},
$$




$$
\wp_{I}(s)=\frac{\pi}{2 \omega_{1}} \frac{\vartheta_{2} \vartheta_{3} \vartheta_{4}}{\vartheta_{I+1}} \frac{\vartheta_{I+1}(u)}{\vartheta_{1}(u)}
$$

where

$$
x=\pi \vartheta_{3}^{2} u=\sqrt{e_{1}-e_{3}} s .
$$

\section{References}

[1] O. Aharony, O. Bergman, D. L. Jafferis and J. Maldacena, JHEP 0810 (2008) 091 arXiv:0806.1218 [hep-th]].

[2] S. Terashima, JHEP 0808 (2008) 080 [arXiv:0807.0197 [hep-th]].

[3] J. Gomis, D. Rodriguez-Gomez, M. Van Raamsdonk and H. Verlinde, JHEP 0809 (2008) 113 [arXiv:0807.1074 [hep-th]].

[4] S. Terashima and F. Yagi, JHEP 0912 (2009) 059 [arXiv:0909.3101 [hep-th]].

[5] S. Terashima and F. Yagi, JHEP 1103 (2011) 036 [arXiv:1012.3961 [hep-th]].

[6] A. Basu and J. A. Harvey, Nucl. Phys. B 713 (2005) 136 hep-th/0412310.

[7] D. Nogradi, JHEP 0601 (2006) 010 hep-th/0511091].

[8] K. Hanaki and H. Lin, JHEP 0809 (2008) 067 arXiv:0807.2074 [hep-th]].

[9] T. Nosaka and S. Terashima, Phys. Rev. D 86 (2012) 125027 arXiv:1208.1108 [hep-th]].

[10] D. -E. Diaconescu, Nucl. Phys. B 503 (1997) 220 hep-th/9608163.

[11] A. Gustavsson, JHEP 0804 (2008) 083 arXiv:0802.3456 [hep-th]].

[12] J. Bagger and N. Lambert, JHEP 0802 (2008) 105 arXiv:0712.3738 [hep-th]].

[13] J. Bagger and N. Lambert, Phys. Rev. D 77 (2008) 065008 arXiv:0711.0955 [hep-th]].

[14] J. Bagger and N. Lambert, Phys. Rev. D 75 (2007) 045020 hep-th/0611108].

[15] A. Gustavsson, Nucl. Phys. B 811 (2009) 66 [arXiv:0709.1260 [hep-th]].

[16] N. J. Hitchin, Commun. Math. Phys. 89 (1983) 145. 
[17] N. Ercolani and A. Sinha, Commun. Math. Phys. 125 (1989) 385.

[18] N. S. Manton and P. Sutcliffe, "Topological Solitons," Cambridge, UK: Univ. Pr. (2004) 493 pp.

[19] D. Harland, S. Palmer and C. Sämann, JHEP 1210 (2012) 167 arXiv:1204.6685 [hep-th]].

[20] S. Novikov, S. V. Manakov, L. P. Pitaevsky and V. E. Zakharov, "Theory of Solitons: The Inverse Scattering Method," New York, USA: Consultants Bureau (1984) 276 pp. ( Contemporary Soviet Mathematics)

[21] O. Babelon, D. Bernard and M. Talon, "Introduction to Classical Integrable Systems," Cambridge, UK: Univ. Pr. (2003) 616 pp.

[22] R. S. Ward, Phys. Lett. A 112 (1985) 3.

[23] P. M. Sutcliffe, Phys. Lett. B 381 (1996) 129 hep-th/9605192. 